\title{
A Novel low power 2-D to 3-D Array Priority Encoder using Split-Logic technique for Data Path Applications
}

\author{
D. S. SHYLU SAM ${ }^{1 *}$, P. SAM PAUL ${ }^{2}$, JENNIFER ELIZAH $^{3}$, NITHYASRI $^{4}$, SNEHITHA $^{5}$, \\ AKANSHA SINGH ${ }^{6}$, VIJENDRA ${ }^{7}$ \\ ${ }_{1,3,4,5,6}$ ECE Department,Karunya Institute of Technology \& Sciences, Coimbatore, INDIA \\ ${ }^{2}$ Mechanical Department, Karunya Institute of Technology \& Sciences, Coimbatore, INDIA \\ ${ }^{7}$ CG Coreel Technologies Pvt. Ltd., Bangalore, INDIA
}

\begin{abstract}
In this work, an ascendable low power 64-bit priority encoder is designed using a two-directional array to three-directional array conversion, and Split-logic technique and 6-bit is obtained as the output. By using this method, the high performance priority encoder can be achieved. In the conventional priority encoder, a single bit is set as an input, but for a priority encoder with 3-Darray, every input are specified in the matrix form. The I-bit input file is split hooked on $\mathrm{M} \times \mathrm{N}$ bits, similar to 2-D Matrix. In priority encoder with 3Darray, three directional output comes out, unlike traditional priority encoder, where the output is received from one direction. The development can be achieved by implementing the two-directional array to threedirectional array technique. Simulation results show that the proposed 2-D and 3-D priority encoder consumes $0.087039 \mathrm{~mW}$ and $0.184014 \mathrm{~mW}$ which is less when compared with the conventional priority encoder. The priority encoders are simulated and synthesized using VHDL in Xilinx Vivado version 2019.2 and the Oasys synthesis tool.
\end{abstract}

Key-Words: - Priority Encoder, split-based technique, low power, Synthesis, HDL,Netlist

Received: March 14, 2021. Revised: October 20, 2021. Accepted: December 15, 2021. Published: January 7, 2022.

\section{Introduction}

A Priority encoder is an algorithm or a circuit that compresses a larger number of binary inputs into a smaller number of outputs[1-2] The inputs to the encoder are $2^{\mathrm{n}}$ bits inputs and produces n-bit output. The output of the priority encoder number starts from zero to the most significant input bit. The performance of the priority encoder increases rapidly, especially for processing a high number of input bits[3-4]. The outputs address can be achieved as binary arrangement, from where parallel data can be regained suitably.

As the computer system and technology became faster, the need for transportable electronics encourages the progress of the power consumption in the Priority encoder structure[5-6]. Therefore, the performance of a priority encoder is increasing speedily since input size is also increased by any numbers of bits[7].

Therefore, certain principles suggest covering the 2-D to 3-D conversion depending on priority encoder, to construct an ascendable highperformance Priority encoder. It mainly emphasizes a methodology to build $2^{\mathrm{n}}$ input bit that is 64-bit and larger sized Priority encoder can be designed based on this technique.

\section{Problem Formulation}

To decrease the consumption of power in electronic devices, keyboard applications, robotic arm control, ship movement ,etc. several methods were suggested in the literature. In parallel priority look ahead 64-bit priority encoder, there are 64 sets of inputs that are distributed as 8 sets, that is $8 \times 8=64$. The 64 sets are given as input to eight OR gates that are aligned parallel to each other and also 8:1 multiplexer which gives 8-bit output. The outputs of the OR gate and the multiplexer is given as input to the $8: 3$ priority encoder, and the outputs of the priority encoder is then combined to give out 6-bit output, using this method power consumption can be reduced. This architecture is used to decrease the Propagation delay and it is used for low and high priority estimation with a flexible structure. The low power and high speed achieved by the priority encoder design when compared to the power consumed by the domino dynamic [8-9] circuit is 
more at unwanted redundant power switching at various dynamic power nodes.

In this paper [10-11] multi-level look-ahead technique is implemented to reduce the consumption of power and also to increase the speed of the circuit. The full parallel priority encoder is designed as it would upsurge the performance of the circuit. One of the other approaches is GDI [12-13] which is also used in the reduction of power consumption. The performance of the circuit depends upon the factors such as delay and power; these factors are decreased during the optimization of the circuit done by executing the latest circuit that includes priority look-ahead parallel architecture [14-15].

The copious quantity of bits is distributed from which only the needed bits are sent as inputs. For example, 1,024 bits are separated into eight 128 bits and procedures. Similarly, the data that is the form of the matrix are stocked in recollection and computed in this work. In multi-match, all the inputs given to the priority encoder, that is 64 bits are then craved up into eight sets each containing 8-bits. As per the split-logic technique, these groups are given as input to the 8-input OR gates. Therefore, eight OR gates deposited parallel to each other, generating eight-bit output. In the architecture of a 64-input priority encoder, the whole of 64 inputs is distributed as smaller sets and every set contains eight bits. So, OR gates with eight inputs are aligned in parallel to each other and gives 8 single-bit outputs. These outputs are given as input to the eight into three priority encoders, causing three outputs. An eight-input MUX circuit is also used to take inputs that are applied for OR gates and these 8 outputs are the inputs to eight into three priority encoders, which further gives out three outputs. Therefore, combining the priority encoders gives six-bit outputs.

\section{Problem Solution}

\subsection{2-D array Priority Encoder}

In the 64:6 2-D array priority encoder, there are 64 inputs. The 64 bits are given to the input of $8: 1$ OR gates as OR0, OR1, up to OR7, in such a way the gates are parallel to each other generating 8 - bit outputs. The input of the 8 -input priority encoder is derived from the output of 8:1input OR gates and generates 3-bit output. The 64 bits' inputs are also given to the 8-8:1 multiplexer and give eight 1-bit of output, which is further sent to the priority encoder with 8 inputs and 3 outputs. The MUX takes the selection lines from the output of the 8-input OR gate. By combining both the priority encoder of 3bit outputs, the final output with 6-bits is generated. Fig. 1 shows the circuit diagram of the 2-D array priority encoder.

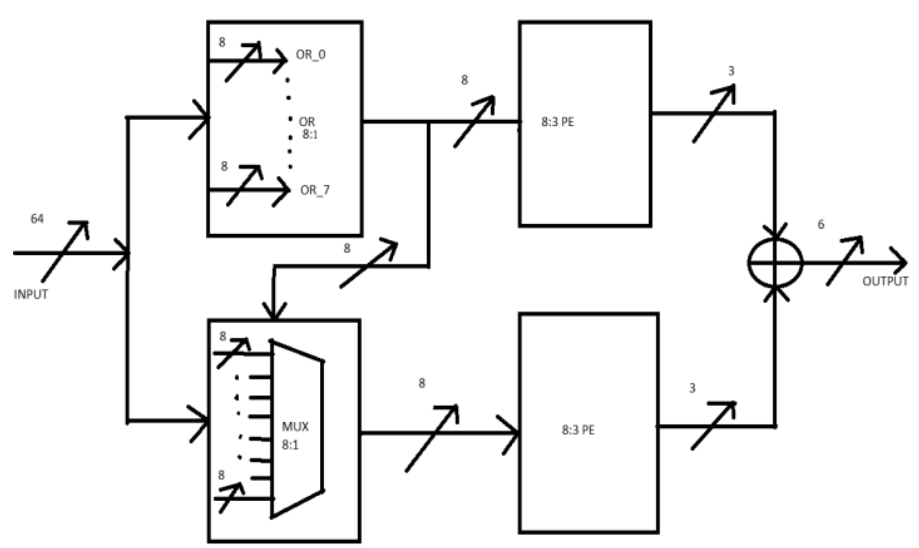

Fig. 1: Circuit of 2-D array priority encoder $64: 6$ 
Table 1. Truth Table of 16X4 Priority Encoder

\begin{tabular}{|c|c|c|c|c|c|c|c|c|c|c|c|c|c|c|c|c|c|c|c|}
\hline \multicolumn{16}{|c|}{ Inputs } & \multicolumn{4}{|c|}{ Outputs } \\
\hline D15 & D14 & D13 & D12 & D11 & D10 & D9 & D8 & D7 & D6 & D5 & D4 & D3 & D2 & D1 & D0 & Q3 & $\mathbf{Q 2}$ & Q1 & Q0 \\
\hline 0 & 0 & 0 & 0 & 0 & 0 & 0 & 0 & 0 & 0 & 0 & 0 & 0 & 0 & 0 & 1 & 0 & 0 & 0 & 0 \\
\hline 0 & 0 & 0 & 0 & 0 & 0 & 0 & 0 & 0 & 0 & 0 & 0 & 0 & 0 & 1 & $\mathrm{x}$ & 0 & 0 & 0 & 1 \\
\hline 0 & 0 & 0 & 0 & 0 & 0 & 0 & 0 & 0 & 0 & 0 & 0 & 0 & 1 & $\mathrm{x}$ & $\mathrm{x}$ & 0 & 0 & 1 & 0 \\
\hline 0 & 0 & 0 & 0 & 0 & 0 & 0 & 0 & 0 & 0 & 0 & 0 & 1 & $\mathrm{x}$ & $\mathrm{X}$ & $\mathrm{X}$ & 0 & 0 & 1 & 1 \\
\hline 0 & 0 & 0 & 0 & 0 & 0 & 0 & 0 & 0 & 0 & 0 & 1 & $\mathrm{x}$ & $\mathrm{x}$ & $\mathrm{x}$ & $\mathrm{X}$ & 0 & 1 & 0 & 0 \\
\hline 0 & 0 & 0 & 0 & 0 & 0 & 0 & 0 & 0 & 0 & 1 & $\mathrm{x}$ & $\mathrm{x}$ & $\mathrm{x}$ & $\mathrm{x}$ & $\mathrm{x}$ & 0 & 1 & 0 & 1 \\
\hline 0 & 0 & 0 & 0 & 0 & 0 & 0 & 0 & 0 & 1 & $\mathrm{x}$ & $\mathrm{x}$ & $\mathrm{x}$ & $\mathrm{x}$ & $\mathrm{x}$ & $\mathrm{x}$ & 0 & 1 & 1 & 0 \\
\hline 0 & 0 & 0 & 0 & 0 & 0 & 0 & 0 & 1 & $\mathrm{X}$ & $x$ & $x$ & $\mathrm{X}$ & $\mathrm{X}$ & $\mathrm{X}$ & $\mathrm{x}$ & 0 & 1 & 1 & 1 \\
\hline 0 & 0 & 0 & 0 & 0 & 0 & 0 & 1 & $\mathrm{X}$ & $\mathrm{X}$ & $\mathrm{x}$ & $\mathrm{x}$ & $\mathrm{X}$ & $\mathrm{X}$ & $\mathrm{X}$ & $\mathrm{X}$ & 1 & 0 & 0 & 0 \\
\hline 0 & 0 & 0 & 0 & 0 & 0 & 1 & $\mathrm{x}$ & $\mathrm{X}$ & $\mathrm{X}$ & $\mathrm{x}$ & $\mathrm{x}$ & $\mathrm{x}$ & $\mathrm{x}$ & $\mathrm{x}$ & $\mathrm{x}$ & 1 & 0 & 0 & 1 \\
\hline 0 & 0 & 0 & 0 & 0 & 1 & $\mathrm{x}$ & $\mathrm{x}$ & $\mathrm{X}$ & $\mathrm{X}$ & $\mathrm{x}$ & $\mathrm{x}$ & $\mathrm{X}$ & $\mathrm{X}$ & $\mathrm{X}$ & $\mathrm{X}$ & 1 & 0 & 1 & 0 \\
\hline 0 & 0 & 0 & 0 & 1 & $\mathrm{x}$ & $\mathrm{x}$ & $\mathrm{x}$ & $\mathrm{x}$ & $\mathrm{x}$ & $\mathrm{X}$ & $\mathrm{x}$ & $\mathrm{x}$ & $\mathrm{X}$ & $\mathrm{X}$ & $\mathrm{X}$ & 1 & 0 & 1 & 1 \\
\hline 0 & 0 & 0 & 1 & $\mathrm{X}$ & $\mathrm{x}$ & $\mathrm{x}$ & $\mathrm{x}$ & $\mathrm{X}$ & $\mathrm{X}$ & $\mathrm{X}$ & $\mathrm{X}$ & $\mathrm{x}$ & $\mathrm{X}$ & $\mathrm{X}$ & $\mathrm{x}$ & 1 & 1 & 0 & 0 \\
\hline 0 & 0 & 1 & $\mathrm{X}$ & $\mathrm{x}$ & $\mathrm{x}$ & $\mathrm{X}$ & $\mathrm{x}$ & $\mathrm{X}$ & $\mathrm{X}$ & $\mathrm{X}$ & $\mathrm{X}$ & $\mathrm{X}$ & $\mathrm{x}$ & $\mathrm{x}$ & $\mathrm{X}$ & 1 & 1 & 0 & 1 \\
\hline 0 & 1 & $\mathrm{X}$ & $\mathrm{x}$ & $\mathrm{x}$ & $\mathrm{x}$ & $\mathrm{x}$ & $\mathrm{x}$ & $\mathrm{x}$ & $\mathrm{x}$ & $\mathrm{X}$ & $\mathrm{X}$ & $\mathrm{x}$ & $\mathrm{x}$ & $\mathrm{x}$ & $\mathrm{x}$ & 1 & 1 & 1 & 0 \\
\hline 1 & $\mathrm{X}$ & $\mathrm{x}$ & $\mathrm{x}$ & $\mathrm{x}$ & $\mathrm{x}$ & $\mathrm{x}$ & $\mathrm{x}$ & $\mathrm{x}$ & $\mathrm{x}$ & $\mathrm{X}$ & $\mathrm{x}$ & $\mathrm{X}$ & $\mathrm{X}$ & $\mathrm{X}$ & $\mathrm{X}$ & 1 & 1 & 1 & 1 \\
\hline
\end{tabular}

\subsection{3-D Array Architecture}

The 64:6 3-D priority encoder has different subblocks, that contain OR gates, Priority encoders, and multiplexers. 64 inputs are given and these 64 inputs are divided into 4-bit as 16 groups, where each group consists of a total of $16 \times 4=64$-bits. OR_A block contains 16-four input OR gates, parallel to each other, giving out 16 single bit outputs where each OR gates consists of 4-bit input and generates one single output. The sixteen single- bit output coming from the OR_A block is given to

OR_B and PE_B blocks. PE_A block consists of 16 priority encoders as $4: \overline{2}$, and are placed corresponding to one another. The output of PE A is 32-bit which is given as input to MUX B. OR B block which takes input from OR_A, consists of 16inputs placed in parallel with 4-bit to each OR gate and gives out 4 single bit outputs. And this four-bit output is given as input to $4: 2$ priority encoder and the output of this PE 4:2 is given as selection lines to MUX_A, MUX_B.

$$
\begin{aligned}
& x=a_{0}+a_{1}+a_{2}+a_{3}+a_{4}+a_{5}+ \\
& a_{6}+a_{7}
\end{aligned}
$$

$$
\begin{aligned}
& y=D_{0} S_{0 \text { bar }} S_{1 \text { bar }} S 2_{\text {bar }}+ \\
& D_{1} S_{0 b a r} S_{1 b a r} S_{2}+D_{2} S_{0 b a r} S_{1} S_{2 b a r}+ \\
& D_{3} S_{0 b a r} S_{1} S_{2}+D_{4} S_{0} S_{1 \text { bar }} S_{2 \text { bar }}+ \\
& D_{5} S_{0} S_{1 \text { bar }} S_{2}+D_{6} S_{0} S_{1} S_{2 \text { bar }}+ \\
& D_{7} S_{0} S_{1} S_{2}
\end{aligned}
$$

$Q_{\text {oTop }}=\sum\left(x_{6 b a r}+x_{\text {fourbar }} x_{2 b a r} x_{1}+\right.$ $\left.x_{4 b a r} x_{3} x_{5}\right)+x_{7}$

$$
\begin{aligned}
& Q_{1 T o p}=\sum\left(x_{5 b a r} x_{4 b a r}\left(x_{\text {two }}+x_{\text {three }}\right)+x_{6}+\right. \\
& x_{7}
\end{aligned}
$$

$$
\begin{aligned}
& Q_{2 \text { Top }}=\Sigma\left(x_{4}+x_{5}+x_{6}+x_{6}\right) \\
& Q_{0 \text { Bottom }}=\sum\left(y_{6 b a r}+y_{\text {fourbar }} y_{2 b a r} y_{1}+\right. \\
& \left.y_{4 b a r} y_{3} y_{5}\right)+y_{7}
\end{aligned}
$$

$$
\begin{aligned}
& Q_{1 \text { Bottom }}=\Sigma\left(y_{5 b a r} y_{4 b a r}\left(y_{\text {two }}+x y_{\text {three }}\right)+\right. \\
& y_{6}+y_{7}
\end{aligned}
$$

$$
\begin{aligned}
& Q_{2 \text { Bottom }}=\Sigma\left(y_{4}+y_{5}+y_{6}+y_{7}\right) \\
& \text { Output }=Q_{\text {top }}+Q_{\text {Bottom }}
\end{aligned}
$$


The input of PE_B block which also takes inputs from output of OR_A, consists of 4 priority encoders as 4:2 and are placed corresponding to each other and develops eight-bit output, which is given as input to MUX_A.MUX_A consists of two 2:1mux circuits and generates 2 bit output. MUX_B consists of two 8:1 MUX circuits and generates $2 \bar{b}$ it output. The output of 4:2 PE, MUX A , and MUX_B are combined to get the final output. Fig.2 shows the circuit diagram of the 3-D priority encoder.

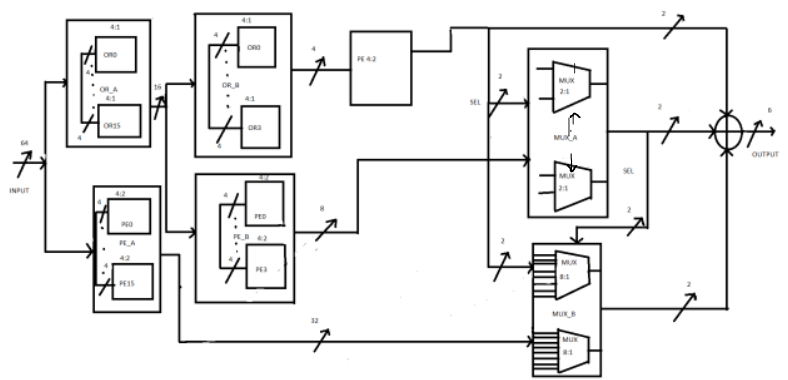

Fig. 2: Shows the circuit diagram of the 3-D priority encoder.

The internal design of the 4:1 MUX circuit is shown in Fig.3. The design generates two outputs and has six 2:1 MUX and has two selection lines. The inputs given to four $2 \mathrm{X} 1$ multiplexer is given as the inputs to $4 \mathrm{X} 1$ multiplexer. The output of the $2 \mathrm{X} 1$ multiplexer is given as inputs to two $2 \mathrm{X} 1$ multiplexer and the $2 \mathrm{X} 1$ multiplexer (q5) selects the inputs from $\mathrm{q} 4$ and provide the output as $\mathrm{q} 2$ and $\mathrm{q} 3$.

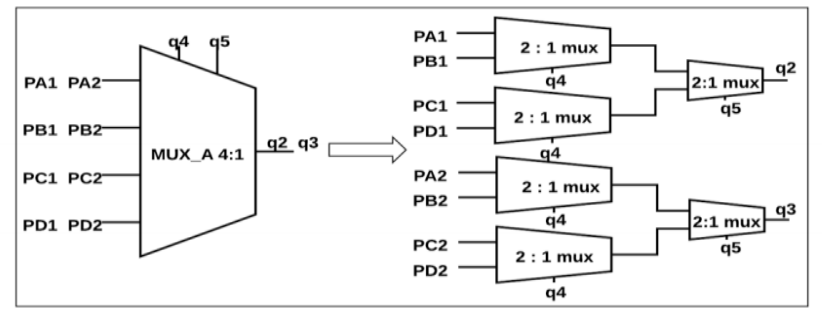

Fig. 3: Internal circuit of 4:1 MUX[2]

The internal circuit 16:1 MUX design is shown in Fig.4. The design generates two outputs has four 2:1 MUX and two 2:1 MUX and has two selection lines. The internal circuit of multiplexer and is used to select from the multiple inputs to a single input.

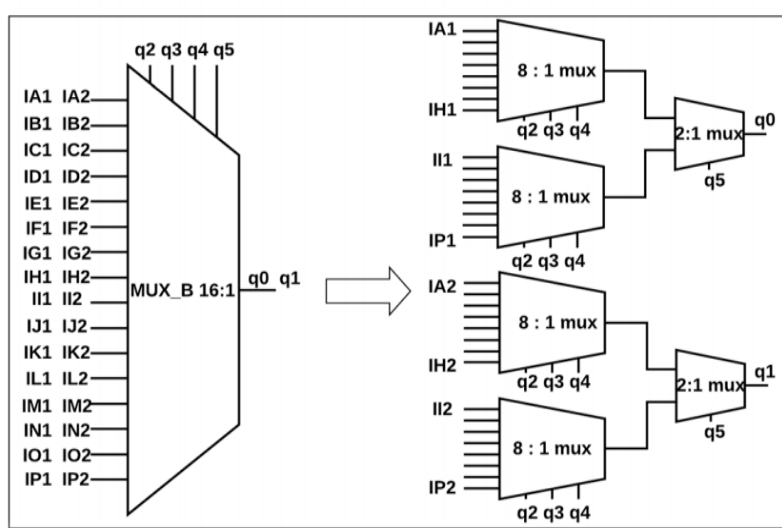

Fig. 4: Internal circuit of 16:1 MUX[2]

Power consumption is one of the essential factors in modern electronics [16-21]. But the expected power cannot be achieved due to the supply voltage, Capacitance, and input operating frequency. As the power consumption is affected by leakage current and other factors it is necessary to reduce the power consumption. The Priority encoder uses priority stage at each input. When there are multiple inputs priority encoder comes into picture and selects the high priority input and all other low priority inputs will be neglected. Hence power consumption is important in 64-bit priority encoder when used for data path applications.

\section{Results and Discussion}

The various logics are splitted and integrated to realize the 2-D and 3-D priority encoder using the Split-logic technique. The input of the 8-input priority encoder is derived from the output of 8:1input OR gates and generates 3-bit output. The 64 bits inputs are also given to the 8-8:1 multiplexer and give eight 1-bit of output, which is further sent to the priority encoder with 8 inputs and 3 outputs. The MUX takes the selection lines from the output of the 8-input OR gate. By combining both the priority encoder of 3-bit outputs, the final output with 6-bits is generated. The 64:6 3-D priority encoder has different sub-blocks, that contain OR gates, Priority encoders, and multiplexers. 64 inputs are given and these 64 inputs are divided into 4-bit as 16 groups, where each group consists of a total of $16 \times 4=64$-bits. The Simulation results are obtained using the Xilinx Vivado tool and synthesis reports are obtained using the Oasys synthesis tool. The power obtained from the designed priority encoder is very less when compared with the conventional priority encoder. Fig. 5,6 shows the schematic of 
block design of 2-D array using Oays synthesis tool and Xilinx Vivado tool.

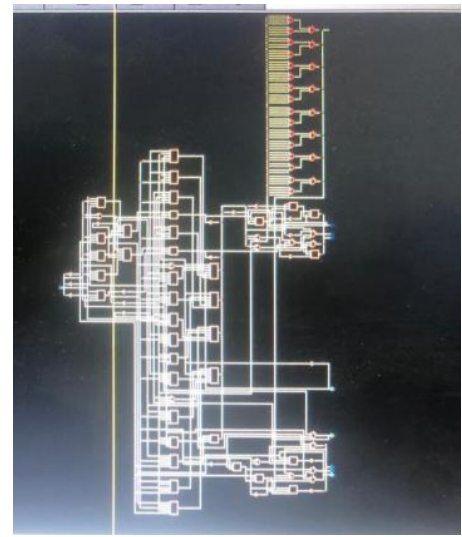

Fig. 5: Block design of 2-D array 64:6 using Oasys synthesis tool

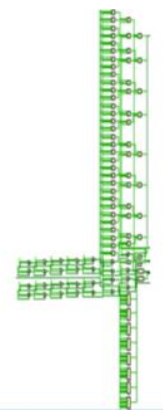

Fig. 6: Block design of 2-D array 64:6

Fig. 7 shows the waveform of the 2-D array priority encoder. The output waveform is verified as per the truth table of the priority encoder. The main advantage of this type of priority encoder is used for complex data path elements. Fig. 8 shows the netlist generated for the 2-D array encoder.

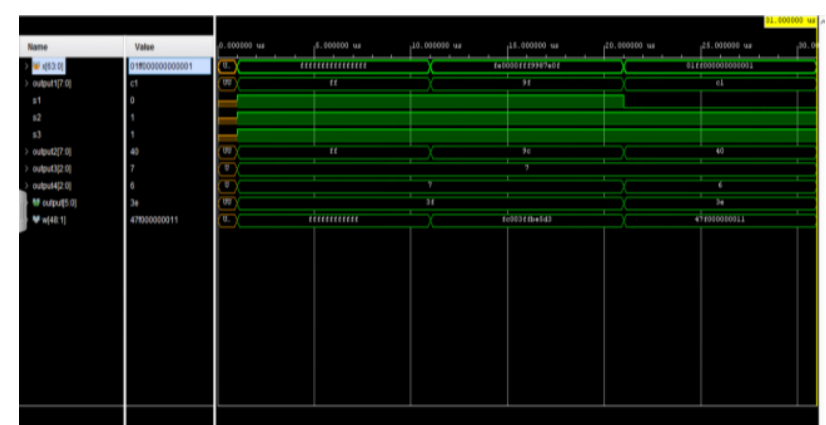

Fig. 7: Waveform of 2-D array 64:6 encoder.

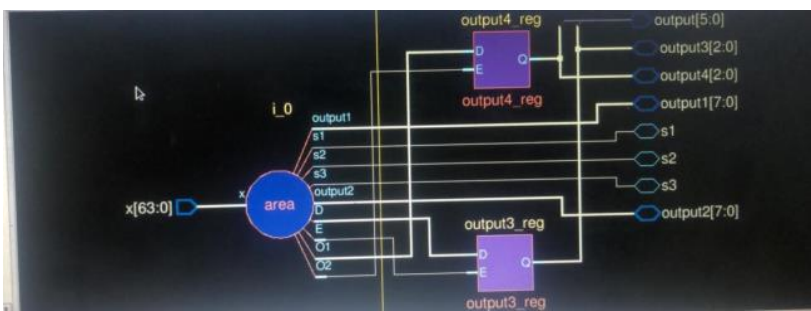

Fig. 8: Netlist generated for 2-D array encoder.

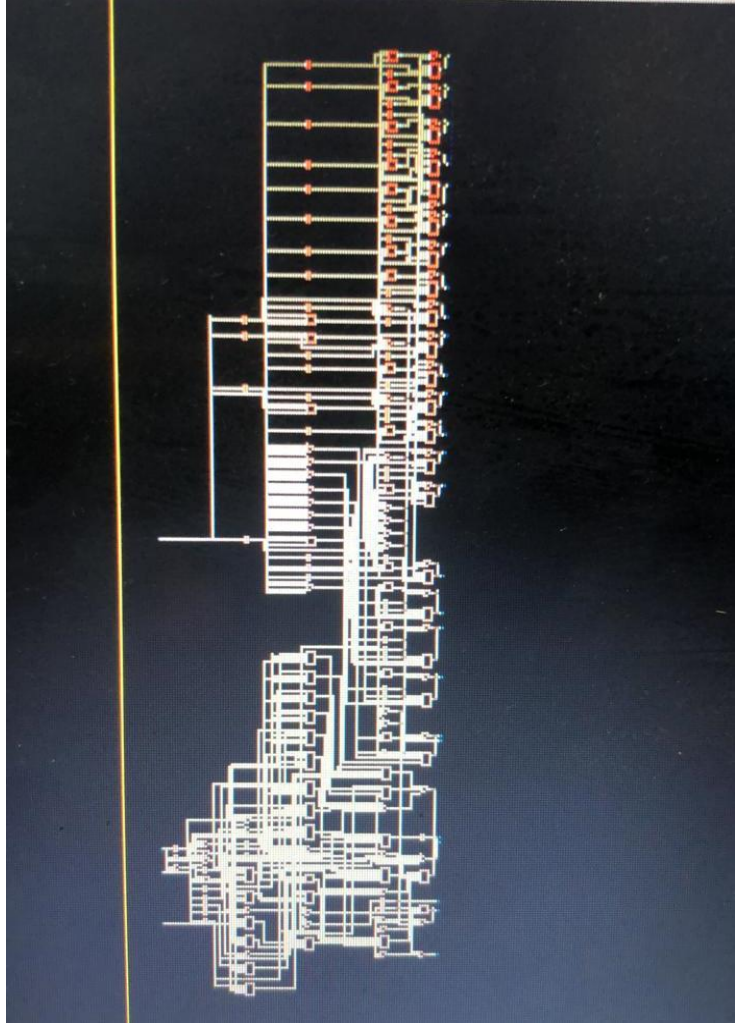

Fig. 9: Block diagram of 3-D array 64:6 using Oasys synthesis tool

Fig. 9,10 shows the block diagram of the 3-D array priority encoder. All the sub-blocks including Priority encoder, OR gate, and Multiplexer are integrated to realize the 3-D array priority encoder.

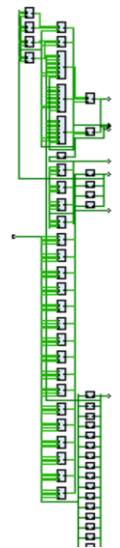

Fig. 10: Block design of 3-D array 64:6encoder

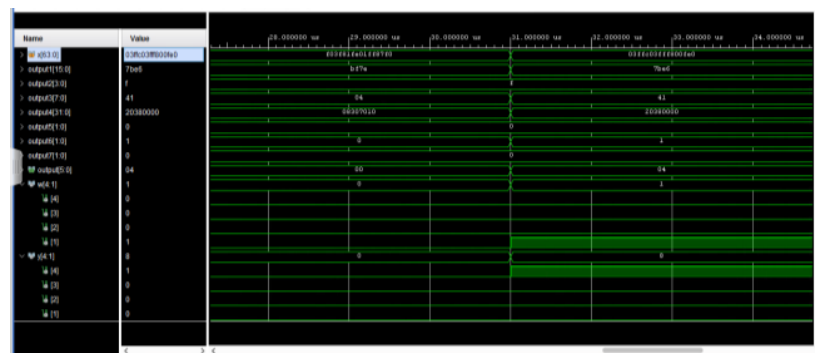

Fig. 11: Waveform of 3-D array 64:6 encoder

Fig. 11 shows the waveform of the 3-D array priority encoder. The output of the 3-D priority 
encoder is verified as per the truth table. Fig. 12 shows the power analysis of the 2-D priority encoder using the Oasys synthesis tool. The total power consumption obtained by the 2-D priority encoder is $0.87039 \mathrm{~mW}$.

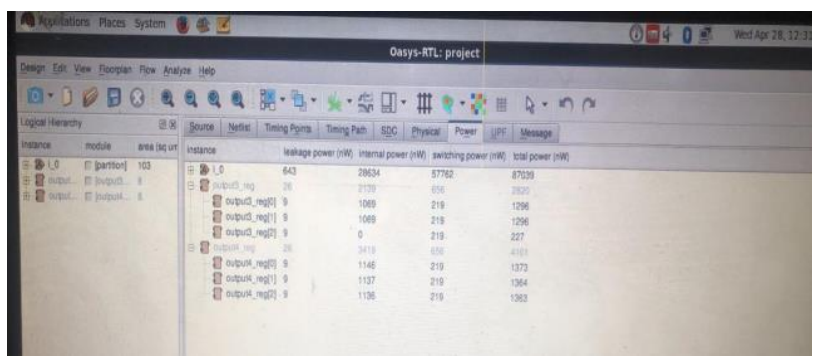

Fig. 12: Power analysis of 2-D using Oasys synthesis tool.

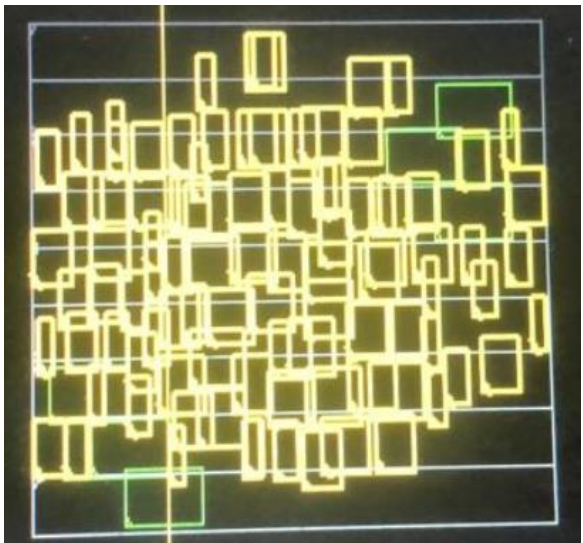

Fig. 13: Physical Verification of 2-D priority encoder

Fig. 13 shows the physical verification of the priority encoder. It shows the interconnected subblocks to realize the 2-D priority encoder. Fig. 14 shows the physical verification of the 2-D priority encoder with all the internal blocks.

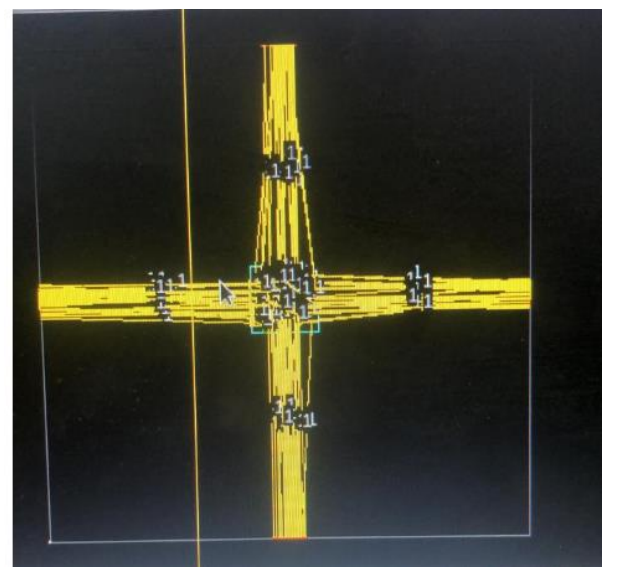

Fig. 14: Physical verification with all the logic subblocks.

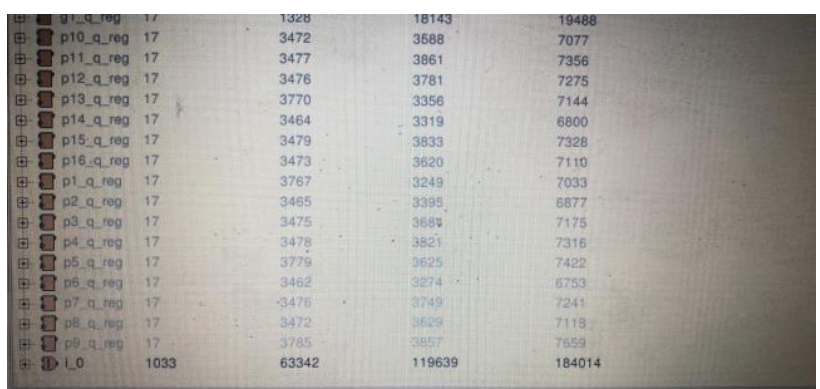

Fig. 15: Power analysis of 3-D array using Oasys Synthesis tool.

Fig. 15 shows the power analysis of the 3-D array using the Oasys synthesis tool. The power obtained for the 3-D priority encoder is $0.184014 \mathrm{~mW}$.

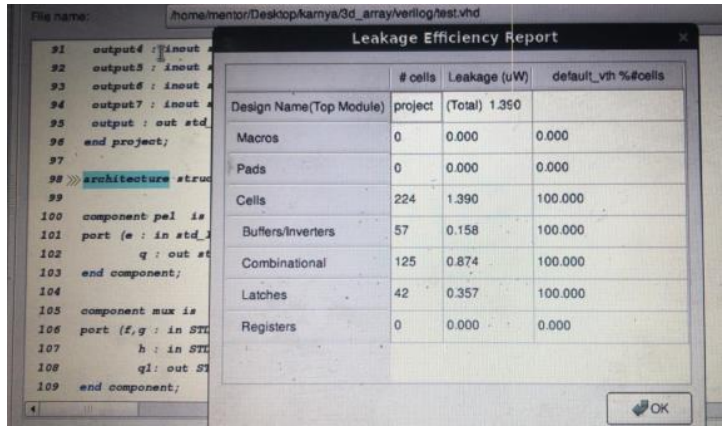

Fig. 16: Leakage efficient report

The leakage power obtained for the 3-D priority encoder is $1.390 \mu \mathrm{W}$. Fig. 16 shows the leakage efficient report. The leakage power of Cells is $1.390 \mu \mathrm{W}$. The leakage power reported for Buffers/Inverters is $0.158 \mu \mathrm{W}$. The leakage power for the combinational circuit is $0.357 \mu \mathrm{W}$. The leakage power of the Latches reported from the synthesis report is $0.357 \mu \mathrm{W}$. Fig. 17 shows the total cell usage report for 3-D priority encoder. In this report the total area $(\mathrm{sq} \mu \mathrm{m})$ is displayed for all the combinational logic. As the power consumption of priority encoder is one of the important factor the leakage power for all combinational logic is given in the report.

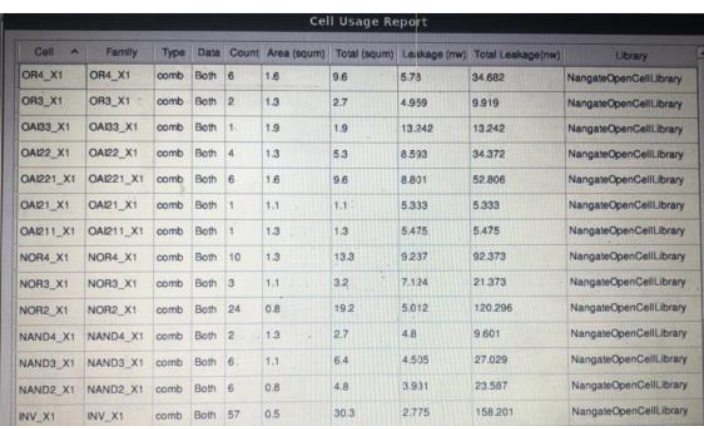

Fig. 17 The total cell usage report for 3-D priority encoder. 


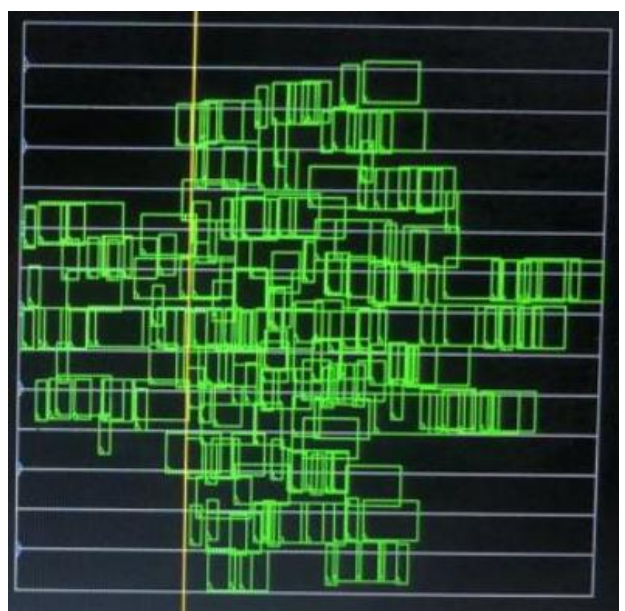

Fig. 18 Physical Verification of 3-D array priority encoder.

Fig.18 shows the Physical Verification of the 3$\mathrm{D}$ array priority encoder. Thus the simulation and synthesis of 2-D and 3-D priority encoder are done and verified. Synthesis results show that the designed priority encoder consumes less power when compared with the existing priority encoders. The designed priority encoder can be used for complex data path elements including adders, multipliers, and Arithmetic Logic Units. Table 1 shows the comparison result of priority encoders with existing work.

Table 1. Comparison result with existing work

\begin{tabular}{|l|l|}
\hline $\begin{array}{l}\text { Reported } \\
\text { Works }\end{array}$ & $\begin{array}{l}\text { Total } \\
\text { Power(mW) }\end{array}$ \\
\hline $\begin{array}{l}\text { 2D-array } \\
\text { 64:6[2] }\end{array}$ & 0.3480 \\
\hline $\begin{array}{l}\text { 3D-array } \\
\text { 64:6[1] }\end{array}$ & 0.2648 \\
\hline $\begin{array}{l}\text { Proposed } \\
\text { 2D-array 64:6 }\end{array}$ & 0.087039 \\
\hline $\begin{array}{l}\text { Proposed } \\
\text { 3D-array 64:6 }\end{array}$ & 0.184014 \\
\hline
\end{tabular}

\section{Conclusion}

The architectures of both the circuits are used for data-path applications and high-speed FLASH ADCs. Simulation results of two dimensional and three-dimensional array priority encoders consume low power consumption. This low power is achieved by using the split-logic technique. The priority encoder with 2 Directional-array consumes $0.87039 \mathrm{~mW}$ and that of priority encoder 3 Directional-array consumes $0.184014 \mathrm{~mW}$. Thus, the power analysis is done and the entire design is simulated and synthesized. Simulation results show that the performance of the proposed priority encoder is high when compared with the conventional encoders. Hence the proposed encoder can be used for data-path applications.

\section{Acknowledgement:}

The authors would like to give sincere thanks to the VLSI Lab of ECE Department, School of Engineering and Technology, Karunya Institute of Technology and Sciences for providing the Mentor Graphics software tool to complete this work.

\section{References:}

[1] K Anil Chandra, N S Murty, "Low Power High Performance Priority Encoder Using 2D-Array to3D-Array Conversion", Procedia computer science 171,2020 , pp.1037-1045.

[2] Nguyen XT., Nguyen HT. and Pham CK,A Scalable High-Performance Priority Encoder Using 1D-array to 2D-array Conversion,IEEE Transactions on Circuits and Systems II: Express Briefs. 64(9),2017,pp.1102-6

[3] Nguyen HT., Nguyen XT. and Pham CK.,An FPGA approach for high-performance multimatch priority encoder, IEICE Electronics Express. 2016,pp.13-20160447.

[4] Mason A., Kun C. and Quan S.,A poweroptimized 64-bit priority encoder utilizing parallel priority look-ahead,IEEE International Symposium on Circuits and System,IEEE Cat. No. 04CH37512,Vol. 2, 2004,pp. II-753.

[5] Abdel-Hafeez S. and Harb S.,A VLSI highperformance priority encoder using standard CMOS library,IEEE Transactions on Circuits and Systems II: Express Briefs,53(8),2006,pp.597-601.

[6] Clark LT. and Maurya SK., Fast and scalable priority encoding using static CMOS, Proceedings of 2010 IEEE International Symposium on Circuits and Systems,2010,pp. 433-436.

[7] Balobas D. and Konofaos N.,Low-power, highperformance 64-bit CMOS priority encoder using static-dynamic parallel architecture". 5th International Conference on Modern Circuits and Systems Technologies (MOCAST),2016, pp. 1-4.

[8] Huang CH, Wang JS, Huang YC. Design of high-performance CMOS priority encoders and incrementer/decrementers using multilevel lookahead and multilevel folding techniques. IEEE Journal of Solid-State Circuits, 37(1), 2002,63-76.

[9] Huang SW, Chang YJ. A full parallel priority encoder design used in comparator,53rd IEEE 
International Midwest Symposium on Circuits and Systems,2010,pp. 877-880.

[10] Sowjith N, Sandeep KS, Sumanth M, Agrawal S. Low power VLSI architecture for combined FMO/Manchester encoder for reusability and FMO/Manchester codecs. In2016 IEEE International Conference on Computational Intelligence and Computing Research (ICCIC) 2016,pp. 1-5.

[11] Maurya SK, Clark LT. A dynamic longest prefix matching content addressable memory for IP routing. IEEE Transactions on Very Large K Anil Chandra et al. / Procedia Computer Science 171 (2020) 1037-1045 1045 K Anil Chandra, N S Murty / Procedia Computer Science 00 (2019) 000-000 9 Scale Integration (VLSI) Systems. 19(6),2010,pp.963-72.

[12] Faezipour M, Nourani M. Wire-speed TCAMbased architectures for multimatch packet classification. IEEE Transactions on Computers,58(1),2008,pp.5-17.

[13] Tambatkar S, Menon SN, Sudarshan V, Vinodhini M, Murty NS. Error detection and correction in semiconductor memories using 3D parity check code with hamming code. In 2017 International Conference on Communication and Signal Processing (ICCSP) 2017,pp. 09740978.

[14] Khaled M. Ali; Hassan Mostafa; Tawfik Ismail. High performance layout-friendly 64-bit priority encoder utilizing parallel priority lookahead.2015 5th International Conference on Energy Aware Computing Systems \& Applications, 2015.

[15] Xuan-Thuan Nguyen; Trong-Thuc Hoang; Hong-Thu Nguyen; Katsumi Inoue; Cong-Kha Pham. "A 219- $\mu \mathrm{W}$ 1D-to-2Dbased priority encoder on 65-nm SOTB CMOS“ , 2018 IEEE International Symposium on Circuits and Systems (ISCAS), 2018.

[16] Manash Pratim Sarma,Kandarpa Kumar Sarma, "A Transmission Gate based High Frequency Rectifier designed using 45nm CMOS Process for RF Energy Harvesting Application." WSEAS Transactions on Circuits and Systems, 18,2019,00.44-49.

[17] D.S. Shylu Sam,Christina (2018) “ Performance Analysis of Array Multiplier using low power 10T Full Adder", Proceedings of the Second International Conference on SCI 2018, Volume 1, Smart Intelligent Computing and Applications,pp.277-285.

[18]Vimukth John,D.S.Shylu,S.Radha,P.Sam Paul,Joel, Design of a power efficient Kogge-
Stone Adder by exploring new OR gate in $45 \mathrm{~nm}$ CMOS Process (CW-12-2018- 0104.R1) in International Journal of Circuit World,2020.

[19]Shylu D.S., Jackuline Moni D., (2016), "Design of low power dynamic comparator with reduced kick back noise using clocked PMOS technique", Journal of Electrical Engineering (JEE), 16(3), 1-10.

[20]Shylu D.S.,Jackuline Moni D. (2014) “A $1.8 \mathrm{~V} 22 \mathrm{~mW} 10$ bit 165 MSPS Pipelined ADC for Video Applications", WSEAS Transactions on Circuits and systems,13, 343-355.

[21] D.S. Shylu Sam, S. Radha, D. Jackuline Moni, P. Sam Paul, J. Jecintha, “ (2019) "Design of 1-V, 12-Bit Low Power Incremental Delta Sigma ADC for CMOS Image Sensor Applications", International Journal of Recent Technology and Engineering (IJRTE) Vol.7, Issue-5S3, 249254.

\section{Creative Commons Attribution License 4.0 (Attribution 4.0 International, CC BY 4.0)}

This article is published under the terms of the Creative Commons Attribution License 4.0 https://creativecommons.org/licenses/by/4.0/deed.en US 